\title{
Restructuration et innovation: une combinaison fascinante
}

\author{
Jan von Overbeck \\ Dr med., Directeur a.i., Services psychiatriques Jura bernois - Bienne-Seeland (SPJBB)
}

Une restructuration est douloureuse, mais présente aussi de grandes opportunités aux collaborateurs de pouvoir construire et redéfinir les activités de l'institution. C'est le cas aux Services psychiatriques Jura bernois - Bienne-Seeland (SPJBB), institution qui sera autonomisée le $1^{\mathrm{er}}$ janvier 2017. Dans ces temps de transition, les SPJBB offrent la possibilité d'associer management et médecine dans un cadre avec un grand potentiel médical.

Les trois institutions psychiatriques du canton de Berne, à savoir les Services psychiatriques universitaires de Berne (SPU), le Centre psychiatrique de Münsingen (CPM) et les Services psychiatriques Jura bernois - BienneSeeland (SPJBB), deviendront autonomes le $1^{\text {er }}$ janvier 2017. Dans ce contexte, les SPJBB doivent repenser toutes leurs activités afin de s'adapter à la nouvelle situation, qui demande une stabilité financière avec des revenus suffisants pour être indépendants. La garantie de déficit disparaîtra au $1^{\mathrm{er}}$ janvier 2017.

Pour atteindre ce but, il est indispensable d'avoir une vision des activités qui soit étroitement liée aux possibilités de revenus pour l'institution. Comme pour de nombreuses entreprises, il est indispensable de revoir les processus de production et d'investiguer les possibilités de nouvelles prestations qui soient à la fois nécessaires pour la santé mentale et générant des revenus. Une des étapes clé est de revoir les attitudes médicales dans l'institution. La psychiatrie a beaucoup évolué ces dernières années et par exemple, les durées d'hospitalisation se raccourcissent, et les soins ambulatoires et les cliniques de jour prennent de l'importance. Le TARPSY sera bientôt introduit et aura des implications dans la prise en charge des patients et sur le financement. Une telle restructuration comporte un mélange fascinant de management et de médecine. Nos activités doivent être de qualité, utiles et nécessaires pour la santé mentale, mais aussi économiques car l'institution doit être financièrement stable. Le réel succès durable est

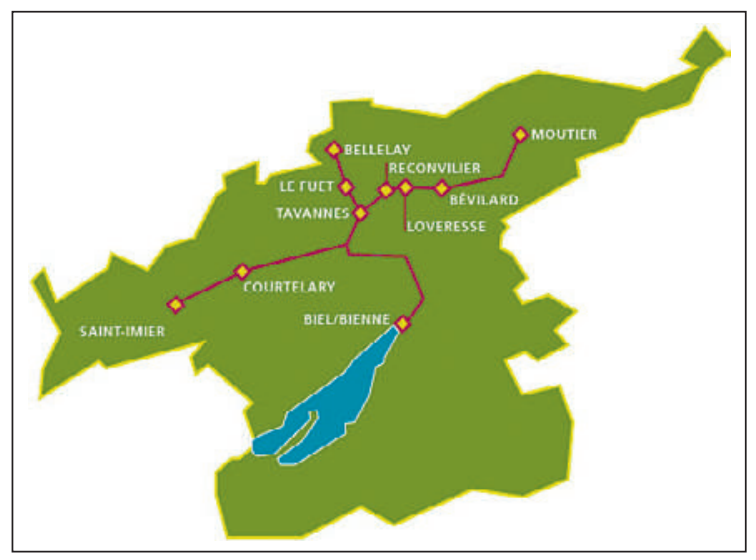

Les Services psychiatriques Jura bernois - Bienne-Seeland (SPJBB).

issu de l'alignement de ces deux aspects fondamentaux et indissociables.

Ces périodes de restructuration et de changement déclenchent de fortes réactions dans les institutions touchées, car chacun craint pour sa place et ses acquis. Cependant, c'est une occasion unique pour repenser toutes les activités et améliorer nos prestations. Une analyse claire et approfondie doit être faite et largement discutée dans l'institution afin de profiter du pool d'idées et d'expériences accumulées. Cette chance d'adapter les structures et notre pensée médicale est unique. Ainsi, la transition d'une institution publique à une SA est certes un challenge important, mais aussi une opportunité unique pour les soignants, car ils peuvent participer et construire leur future activité dans le cadre de la SA. C'est la raison pour laquelle je souhaite, par ce court article, réveiller l'intérêt de mes collègues médecins et infirmiers pour cette activité et possibilité d'apprendre à associer management et médecine, et vous encourager à postuler dans nos services afin de participer à cette transition qui a un très grand potentiel médical. 